\title{
5-ASAs in Crohn's Disease: Time to Stop the Salicylate?
}

\author{
Sailish Honap ${ }^{1,2} \cdot$ Esha Sharma $^{1} \cdot$ Mark A. Samaan $^{1}$
}

Accepted: 21 October 2021 / Published online: 19 November 2021

(C) The Author(s), under exclusive licence to Springer Science+Business Media, LLC, part of Springer Nature 2021

Despite accumulating evidence pointing toward their lack of efficacy, the use of 5-aminosalicylates (5-ASA) in Crohn's disease $(\mathrm{CD})$ remains prevalent, contrary to the recommendations of most national and international guidelines. Cochrane systematic reviews of randomized trials have previously showed no benefit for 5-ASA drugs over placebo for inducing or maintaining remission in $\mathrm{CD}[1,2]$. Notwithstanding the above, 5-ASAs are one of the most commonly prescribed group of drugs in CD. For example, data from a recent retrospective analysis of a primary care database showed that $44.2 \%$ of patients with CD were prescribed a 5-ASA in the UK [3]. Research has focused on whether discontinuing 5-ASAs in those established on immunosuppressive therapy leads to disease exacerbation. Addressing this area has the potential to improve the financial burden on the health economy and reduce patient exposure to unnecessary drugs.

Most patients with $\mathrm{CD}$ will require escalation to an immunomodulatory and/or biologic therapy at some point in their disease course. It is becoming increasingly accepted that continuing 5-ASAs in patients receiving biologics confers no additional benefit for both ulcerative colitis (UC) and CD. In two nationwide population-based cohorts, Ungaro et al. [4] showed that stopping 5-ASA in CD did not increase risk of adverse outcomes (steroid use, hospitalization, or surgery) both in a large US cohort $(n=2960)$ (adjusted hazard ratio [AHR], 0.89; 95\% CI, 0.77-1.03; $\mathrm{P}=0.13$ ) or in a Danish cohort $(n=218)$ (AHR, $1.13 ; 95 \%$ CI, $0.68-1.87 ; \mathrm{P}=0.63)$.

Mark A. Samaan

mark.samaan@gstt.nhs.uk

Sailish Honap

shonap@nhs.net

Esha Sharma

esha.sharma@gstt.nhs.uk

1 IBD Centre, St Thomas' Hospital, NHS Foundation Trust, Westminster Bridge Road, Guy's and St Thomas', London SE1 7EH, UK

2 School of Immunology and Microbial Sciences, King's College London, London, UK
Although no demonstrable benefit of continuing vs stopping 5-ASA has been observed in patients with UC escalated to anti-metabolite therapy, the situation is less clear for CD [5].

In this issue of Digestive Diseases and Sciences, Picetti et al. [6] report the findings of a retrospective analysis of an administrative claims database designed to identify patterns of 5-ASA use in patients with CD in whom therapy with anti-metabolites was newly initiated. The categorization of 5-ASA exposure was based on the number of prescriptions issued and the time interval after anti-metabolite initiation with "stopping" defined as no further prescriptions, "shortterm" for prescriptions issued $<6$ months, and "persistent" 5-ASA use defined as prescriptions issued 6-12 months later. The primary outcomes were new corticosteroid use, escalation to a biologic, CD-related hospitalization, and surgery.

In total, 3036 patients with $\mathrm{CD}$ receiving newly initiated anti-metabolites were included in the analysis. Unsurprisingly, a significant proportion of the studied cohort (57.8\%) had persistent 5-ASA use, reflecting their known widespread use in CD. Reassuringly, 5-ASA discontinuation did not increase the risk of CD-related hospitalization (HR, 1.21 [0.98-1.49]), CD-related surgery (HR, 1.28 [0.90-1.80]) or treatment escalation (HR, 0.85 [0.62-1.20]). Of interest, patients with persistent 5-ASA use had an increased risk of requiring corticosteroids (HR, 1.24; 95\% CI, 1.08-1.42), which as the authors discuss in their article, may simply reflect the fact that 5-ASA was less likely to be stopped in those with severe disease.

While this article is a valuable addition to the literature and complements the findings of a recently published Canadian study examining 5-ASA use in a similar population over two decades, one must interpret these findings with a degree of caution [7]. This was a retrospective observational study, which is limited by confounding factors inherent to this type of study design. Further, important data regarding objective disease severity assessment, CD phenotype, and disease distribution were unavailable, the latter being of particular interest given the previously conflicting evidence 
of the contribution of 5-ASA to the pathogenesis of isolated colonic CD.

The lack of data supporting the utility of 5-ASAs as an adjunct to immunosuppression in $\mathrm{CD}$ is becoming increasingly clear. Accruing data showing no clear or consistent evidence of added efficacy, providing a good rationale for drug discontinuation. There are a number of other potential advantages that should be considered. Firstly, whereas 5-ASAs are low cost compared with advanced therapies used in IBD, the cumulative cost is not insignificant. In the United Kingdom (UK), since mesalamine preparations cost approximately $£ 1000$ per year for those receiving the highest doses, it is a significant cost driver for UC treatment throughout Europe [8]. In a healthcare system aiming to deliver costeffective care, Picetti et al. [4] highlight that a considerable cost saving can be made by stopping 5-ASAs. In some cases, depending on brand, the annual cost of mesalamine may approach that of highly effective therapies, such as biosimilar adalimumab. In addition to the impact on the US insurance-based system, detailed by Picetti and colleagues, these findings are also pertinent for single-payer, nationalized healthcare systems such as the UK. Although funding for numerous treatments has historically been separated into primary (general) and secondary (specialist) care, as healthcare systems move toward greater integration, an overall appreciation for the most cost-effective strategy is imperative. Secondly, the excess pill burden for patients should not be underestimated and may lead to poor adherence to oral therapies, which have greater potential therapeutic benefit. Thirdly, although uncommon, patients are unnecessarily exposed to the risks of GI disturbance and the less common risk of pancreatitis and nephrotoxicity. Finally, initiating a potentially ineffective drug may delay initiating therapies of evident benefit. Despite all of this, 5-ASAs continue to be prescribed for $\mathrm{CD}$ by healthcare practitioners worldwide.

The STopping Aminosalicylate Therapy in Inactive Crohn's Disease (STATIC) study is an open-label, randomized, non-inferiority trial randomizing patients with $\mathrm{CD}$ in remission to either continue 5-ASA therapy or withdraw treatment [9]. Recruitment is ongoing and the results are eagerly awaited. The hypothesized lack of impact of 5-ASA withdrawal on disease course in a prospective, controlled setting, in conjunction with the type of retrospective largescale data presented here by Picetti and colleagues, would no doubt make a compelling argument for discontinuation. Though one hopes that this type of thorough and manifold approach is enough to settle this enduring issue and positively influence practice, this remains to be seen. Nonetheless, it would seem that in $\mathrm{CD}$, the time has come to stop the salicylate.

\section{Declarations}

Conflict of interest Sailish Honap served as a speaker, a consultant, and/or an advisory board member for Pfizer, Janssen, and Takeda. Esha Sharma served as a speaker and/or advisory board member for Takeda and Pharmacosmos. Mark Samaan served as a speaker, a consultant, and/or an advisory board member for Sandoz, Janssen, Takeda, MSD, Falk, Abbvie, Bristol Myers Squibb and Samsung Bioepis.

\section{References}

1. Lim W-C, Wang Y, MacDonald JK, et al. Aminosalicylates for induction of remission or response in Crohn's disease. Cochrane Database of Systematic Reviews [Internet]. 2016 [cited 2021 Sep 26];(7). Available from: https://www.cochranelibrary.com/cdsr/ doi/https://doi.org/10.1002/14651858.CD008870.pub2/full

2. Akobeng AK, Zhang D, Gordon M, et al. Oral 5-aminosalicylic acid for maintenance of medically-induced remission in Crohn's disease. Cochrane Database Syst Rev. 2016 Sep 28;9:CD003715.

3. Hart A, Ng SC, Watkins J et al. The use of 5-aminosalicylates in Crohn's disease: a retrospective study using the UK Clinical Practice Research Datalink. Ann Gastroenterol. 2020;33:500-507.

4. Ungaro RC, Limketkai BN, Jensen CB et al. Stopping Mesalamine Therapy in Patients With Crohn's Disease Starting Biologic Therapy Does Not Increase Risk of Adverse Outcomes. Clin Gastroenterol Hepatol. 2020;18:1152-1160.e1.

5. Singh S, Kim J, Zhu W et al. No benefit of continuing vs stopping 5-aminosalicylates in patients with ulcerative colitis escalated to anti-metabolite therapy. Alimentary Pharmacology \& Therapeutics. 2020;52:481-491.

6. Picetti D, Kim J, Zhu W, et al. No Benefit of Continuing 5-Aminosalicylates in Patients with Crohn's Disease Treated with Antimetabolite Therapy. Dig Dis Sci. (Epub ahead of print). doi: https://doi.org/10.1007/s10620-021-07301-x.

7. Bernstein CN, Tenakoon A, Singh $\mathrm{H}$ et al. Continued 5-ASA use after initiation of anti-TNF or immunomodulator confers no benefit in IBD: a population-based study. Alimentary Pharmacology \& Therapeutics. 2021;54:814-832.

8. Buchanan J, Wordsworth S, Ahmad T et al. Managing the long term care of inflammatory bowel disease patients: The cost to European health care providers. J Crohns Colitis. 2011;5:301-316.

9. Alimentiv Inc. Stopping Aminosalicylate Therapy in Inactive Crohn's Disease (STATIC) Study: A Randomized, Open-label, Non-inferiority Trial [Internet]. clinicaltrials.gov; 2021 Apr [cited 2021 Sep 23]. Report No.: NCT03261206. Available from: https:// clinicaltrials.gov/ct2/show/NCT03261206

Publisher's Note Springer Nature remains neutral with regard to jurisdictional claims in published maps and institutional affiliations. 